\title{
Overexpression of telomerase-associated chaperone proteins in prostatic intraepithelial neoplasia and carcinomas
}

\author{
LYNNE W. ELMORE ${ }^{1,4}$, ROBERT FORSYTHE ${ }^{1}$, HEIDI FORSYTHE ${ }^{1}$, A. TAYLOR BRIGHT ${ }^{1}$, \\ SUHAIL NASIM $^{1}$, KANENDORI ENDO ${ }^{1}$ and SHAWN E. HOLT ${ }^{1-4}$ \\ Departments of ${ }^{1}$ Pathology, ${ }^{2}$ Human Genetics, ${ }^{3}$ Pharmacology and Toxicology, and ${ }^{4}$ Massey Cancer Center \\ Medical College of Virginia at Virginia Commonwealth University, Richmond, VA 23298, USA
}

Received February 28, 2008; Accepted March, 2008

DOI: $10.3892 /$ or_00000049

\begin{abstract}
Over $90 \%$ of prostate cancers express telomerase activity. In an experimental model, hsp90 and p23, which are necessary for telomerase assembly and function, dramatically increase during tumorigenic conversion. We immunohistochemically analyzed 60 prostate carcinomas, 50 prostatic intraepithelial neoplasias (PIN) and 25 benign prostatic tissues to determine whether hsp90/p23 expression correlates with advancing stage and whether chaperone distribution overlaps with hTERT, the catalytic component of telomerase. Strong expression of hsp90/p23 was detected in $\sim 95 \%$ of PIN and carcinomas without relationship to Gleason score. While hsp90/p23 immunostaining was predominantly diffuse and cytoplasmic, nuclear immunoreactivity was observed in several moderate-to-high grade carcinomas, and those carcinomas with nuclear chaperone staining exhibited detectable hTERT. Our data suggest enhanced chaperonemediated telomerase assembly as a mechanism for increased activity in advanced prostate carcinomas, stable association between chaperones and telomerase in vivo, and utility for chaperone immuno-staining to identify focal PIN in the context of widespread hyperplasia.
\end{abstract}

\section{Introduction}

Telomerase is a ribonuclear protein complex that maintains telomeric DNA in the vast majority of human cancers, including $>90 \%$ of prostate carcinomas (1). Human telomerase is minimally comprised of an RNA subunit (hTR) (2) and a reverse transcriptase protein component (hTERT) $(3,4)$. Our in vitro data indicate that proper assembly and function of the

Correspondence to: Dr Shawn E. Holt, Department of Pathology, Virginia Commonwealth University, 1101 East Marshall Street, Richmond, VA 23298-0662, USA

E-mail: sholt3@mcvh-vcu.edu

Key words: chaperones, hsp90, p23, telomerase, hTERT, prostate cancer, prostatic intraepithelial neoplasia telomerase holoenzyme is dependent on the stable association of hsp90 and its co-chaperone, p23 $(5,6)$. Hsp90 is ubiquitously expressed representing $1-2 \%$ of total protein in a non-stressed cell. It binds to and facilitates the proper conformation of several client proteins implicated in carcinogenesis including telomerase, androgen receptor, AKT and HER2 (7). Hsp90 is expressed at high levels in a number of human cancer cell types, thus providing a strong rationale for testing the efficacy of specific inhibitors of hsp90 in clinical trials $(8,9)$.

Using an experimental system of prostate cancer progression, we have previously observed a significant increase in telomerase activity without any detectable change in the levels of either hTR or hTERT (10). Instead, the levels of several hsp90-associated chaperones dramatically increased during tumor progression, indicating enhanced assembly of telomerase as a novel regulatory mechanism. Our previous immunohistochemical analysis of a small set of clinical specimens $(n=20)$ revealed low to undetectable expression of hsp90 and p23 in benign prostatic hyperplasia (BPH), which is characterized by relatively low telomerase activity (11), and high levels of co-expression in prostate carcinomas, hinting that expression of the hsp90 chaperone complex increases as a function of disease state in clinical prostate specimens (10). Two studies using relatively large clinical sample sets have subsequently confirmed that expression levels of hsp90 are significantly greater in malignant tissues than in normal prostate tissues $(12,13)$.

The co-chaperone, p23, stabilizes the ATP-bound form of hsp90, facilitating the chaperoning cycle (14). Recent data indicate that p23 may also mediate cellular processes independent of the hsp90 chaperone complex (15). p23 is upregulated in primary and metastatic human lung cancers (15), in a small sample set of prostate carcinomas (10), and during mammary cancer progression (15). We are unaware of any study that has correlated p23 expression levels with advancing stage of prostate cancer or with expression of telomerase components in clinical specimens. Thus, the goal of our study was to analyze a large sample set of prostate tissues, including BPH, PIN and carcinoma, to determine: 1, whether p23 staining intensity correlates with disease state or Gleason score, the strongest predictor of prostate cancer biologic behavior; and 2, if nuclear hsp90 and p23 are detectable in telomerase-expressing prostate cancer cells. 


\section{Materials and methods}

Case selection. Seventy-two formalin-fixed paraffin-embedded specimens, dating back from 1994-2001, were selected from the archives of the Department of Pathology at Virginia Commonwealth University. Sixty-eight were derived from prostatectomy and 4 cases of transurethral resection of the prostate (TURP). The patient age ranged from 49-83 with a mean age of $61.7 \pm 7.7$. The combined Gleason scores of the 64 cases of prostate carcinoma ranged from 5-9 with a median score of 6 . From this case set, we selected 60 carcinomas (average combined Gleason score of 6.5), of which 50 contained PIN (all high grade) and 25 benign prostatic tissues [representing benign prostatic hyperplasia $(\mathrm{BPH})$ and prostatitis].

Immunohistochemistry. The specificity and optimization of the hsp90 and p23 antibodies has been previously reported (10). hTERT antibody specificity was confirmed by Western blotting (data not shown). Antibody-mediated detection was performed using a standard streptavidin-biotin peroxidase complex method (Vector Laboratories, Burlingame, CA). For hsp90 and p23, antigen epitopes were retrieved by a routine steam method using citrate buffer $(\mathrm{pH}$ 6.0), with all subsequent steps being performed on a Dako autostainer. Tissue sections from the representative blocks were deparaffinized in xylene and alcohols and were then incubated with $3 \%$ hydrogen peroxide in a phosphate buffer solution $(\mathrm{pH} \mathrm{7.4)}$ for $10 \mathrm{~min}$ to block endogenous peroxidase activity. hsp90 (1:500; provided by Dr David Toft, Mayo Clinic, Rochester, MN) and p23 (1:5000; JJ3 monoclonal provided by Dr Toft) antibodies were incubated for $30 \mathrm{~min}$ at room temperature. Following secondary antibody incubations, washes, and chromagen (3-3'-diaminobenzidine) detection, slides were counterstained with hematoxylin, dehydrated and mounted for microscopic evaluation. For hTERT immunostaining, antigen retrieval involved autoclaving in citrate buffer at $120^{\circ} \mathrm{C}$ for $30 \mathrm{~min}$ and then allowing the samples to equilibrate to room temperature. Following permeabilization in $0.05 \%$ Tween-20 in PBS for $10 \mathrm{~min}$ at room temperature and quenching of endogenous peroxidase activity, sections were incubated overnight at $4^{\circ} \mathrm{C}$ with hTERT monoclonal $(2.5 \mu \mathrm{g} /$ $\mathrm{ml}$; Alpha Diagnostic, EST21-A). After manual secondary antibody incubations, washes, and chromagen (3-3'-diaminobenzidine) detection, slides were counterstained with hematoxylin, dehydrated and mounted. Negative control slides included omission of the respective antibodies.

Assessment of immunostaining. Immunostaining was evaluated by two independent observers (R.F. and S.N.). Hematoxylin and eosin stained sections were used to confirm tissue diagnosis and Gleason scores. Immunostaining intensity was scored as undetectable (0), weak (1), moderate (2), or intense (3) and relative to histologically non-neoplastic glands with clearly visible basal cell layers. Immunostaining was also scored as nuclear, cytoplasmic or both with a diffuse or focal distribution.

Statistical analysis. $\chi^{2}$ tests were used to study the relationship between hsp90 or p23 expression levels/distribution and
Table I. Elevated hsp90 and p23 overexpression in PIN and carcinoma but not $\mathrm{BPH}$.

\begin{tabular}{llcc}
\hline $\begin{array}{l}\text { Pathology } \\
(\mathrm{n})^{\mathrm{a}}\end{array}$ & Chaperone & $\begin{array}{c}\text { Overexpressed } \\
(\%)\end{array}$ & $\begin{array}{c}\text { Average } \\
\text { intensity }\end{array}$ \\
\hline Benign (25) & hsp90 & 0 & 0.3 \\
& p23 & 0 & 0.3 \\
High grade PIN (50) & hsp90 & 96 & 1.9 \\
& p23 & 94 & 1.8 \\
PrCa (60) & hsp90 & 97 & 1.9 \\
& p23 & 98 & 1.9 \\
\hline
\end{tabular}

${ }^{\mathrm{a}} \mathrm{n}$, number of cases analyzed.

combined Gleason score. A p-value $<0.05$ was considered significant. All statistical analyses were conducted using JMP statistical software (SAS Institute, Inc., Cary, NC).

\section{Results}

The vast majority of prostate carcinomas expressed moderate to high levels of hsp90 and its co-chaperone, p23 (Table I). This was true of adenocarcinomas (Fig. 1) as well as histologic variants such as hypernephroid and mucinous carcinomas (data not shown). Both chaperones were also co-expressed in $\geq 95 \%$ of high grade PIN at an average intensity comparable to that observed in the cancers (Table I and Fig. 1). hsp90 and p23 immunostaining for the cancers (hsp90, 95\%; p23, 93\%) and PIN (hsp90, 92\%; p23, 94\%) was predominantly diffuse. Epithelial cells within the glandular structures of benign glandular structures had undetectable or weak immunoreactivity for both proteins (Table I and Fig. 1). As expected for chaperones that have many client proteins involved in numerous cellular processes (7), hsp90 and p23 were colocalized at moderate to intense levels in many normal prostatic constituents, including ducts, ganglia, seminal vesicles as well as basal cells delineating benign glandular structures and PIN (data not shown).

$\chi^{2}$ tests revealed that $\mathrm{p} 23$ immunostaining intensity did not correlate with Gleason score (Table II) or patient age (data not shown). As reported previously (12), we found that hsp90 immunostaining did not correlate with severity of disease (Table II) or age (data not shown). However, a subset of carcinomas with moderately higher combined Gleason score (mean of 6.9) than the entire sample set (mean of 6.5) was significantly more likely to exhibit nuclear positivity for hsp90 and/or p23 (Table III).

Since these chaperones are necessary for proper telomerase assembly (5) and remain stably associated with telomerase after in vitro assembly (6), we immunohistochemically stained the carcinomas displaying nuclear hsp90 chaperone complex for hTERT. Of the 9 cases analyzed (6/9 expressed nuclear hsp90 and 9/9 expressed nuclear p23), all expressed hTERT (representative staining in Fig. 2), thus 


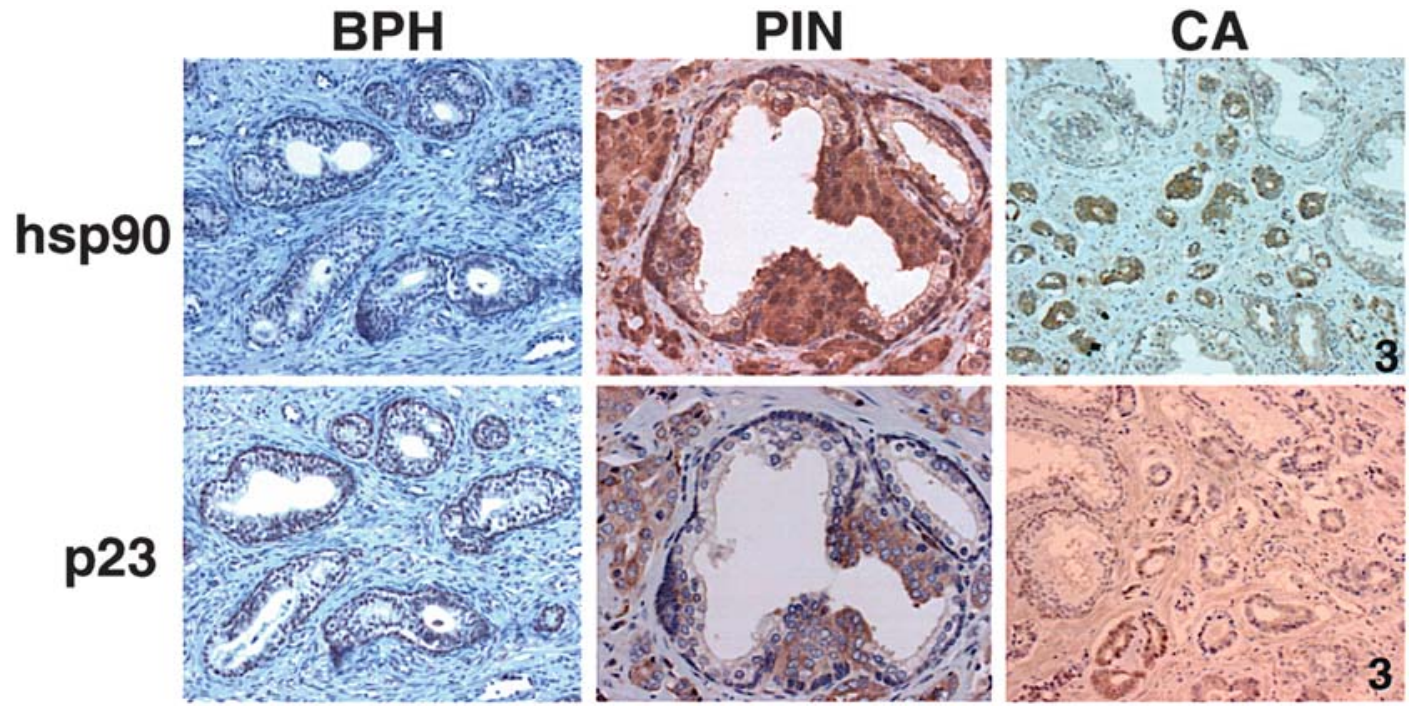

Figure 1. High levels of p23 and hsp90 in PIN and carcinomas but not BPH. Numbers reflect the primary Gleason score.

Table II. hsp90 and p23 immunostaining intensity does not correlate with combined Gleason score.

\begin{tabular}{|c|c|c|c|c|c|}
\hline & \multicolumn{4}{|c|}{ No. hsp90 expression (\%) } & \multirow[b]{2}{*}{ P-value } \\
\hline & 0 & 1 & 2 & 3 & \\
\hline Combined Gleason score & & & & & 0.2429 \\
\hline$\leq 6$ & $1(2.86)$ & $10(28.57)$ & $22(62.86)$ & $2(5.71)$ & \\
\hline 7 & $0(0.00)$ & $5(25.00)$ & $10(50.00)$ & $5(25.00)$ & \\
\hline \multirow[t]{3}{*}{$\geq 8$} & $0(0.00)$ & $\begin{array}{ll}0 & (0.00)\end{array}$ & $3(60.00)$ & $2(40.00)$ & \\
\hline & \multicolumn{4}{|c|}{ No. p23 expression (\%) } & \\
\hline & 0 & 1 & 2 & 3 & P-value \\
\hline Combined Gleason score & & & & & 0.8090 \\
\hline$\leq 6$ & $2(5.71)$ & $9(25.71)$ & $19(54.29)$ & $5(14.29)$ & \\
\hline 7 & $0(0.00)$ & $3(15.00)$ & $12(60.00)$ & $5(25.00)$ & \\
\hline$\geq 8$ & $0(0.00)$ & $1(20.00)$ & $3(60.00)$ & $1(20.00)$ & \\
\hline
\end{tabular}

Table III. Nuclear hsp90 and p23 is associated with a higher combined Gleason score.

\begin{tabular}{|c|c|c|c|c|c|c|}
\hline & \multicolumn{3}{|c|}{ No. hsp90 location (\%) } & \multicolumn{3}{|c|}{ No.p23 location (\%) } \\
\hline & Cytoplasm & Nucleus & P-value & Cytoplasm & Nucleus & P-value \\
\hline Gleason score & & & 0.0291 & & & 0.0172 \\
\hline$\leq 6$ & 34 (97.14) & $1 \quad(2.86)$ & & 33 (94.29) & $2 \quad(5.71)$ & \\
\hline$\geq 7$ & $20(80.00)$ & $5 \quad(20.00)$ & & $18 \quad(72.00)$ & (28) & \\
\hline
\end{tabular}

establishing a strong association between the hsp90 chaperone complex and telomerase in the nuclei of a subset of prostate carcinomas. Glandular epithelial cells of PIN, but not of normal glands, expressed nuclear hTERT, while basal cells supporting both normal glands and carcinoma in situ expressed high levels of hTERT (data not shown), as previously described (16). 

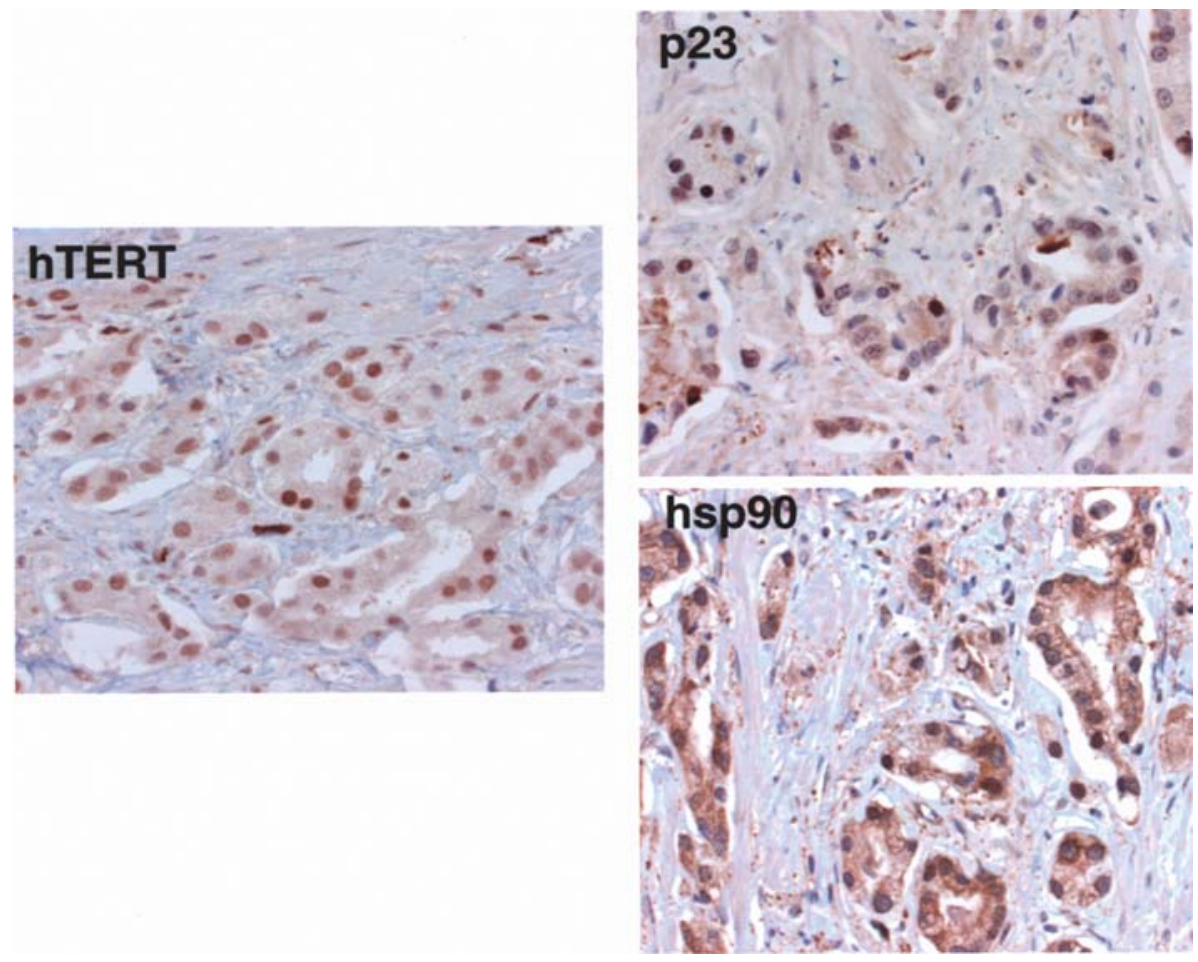

Figure 2. Prominent nuclear staining of hsp90 and p23 correlates with hTERT localization.
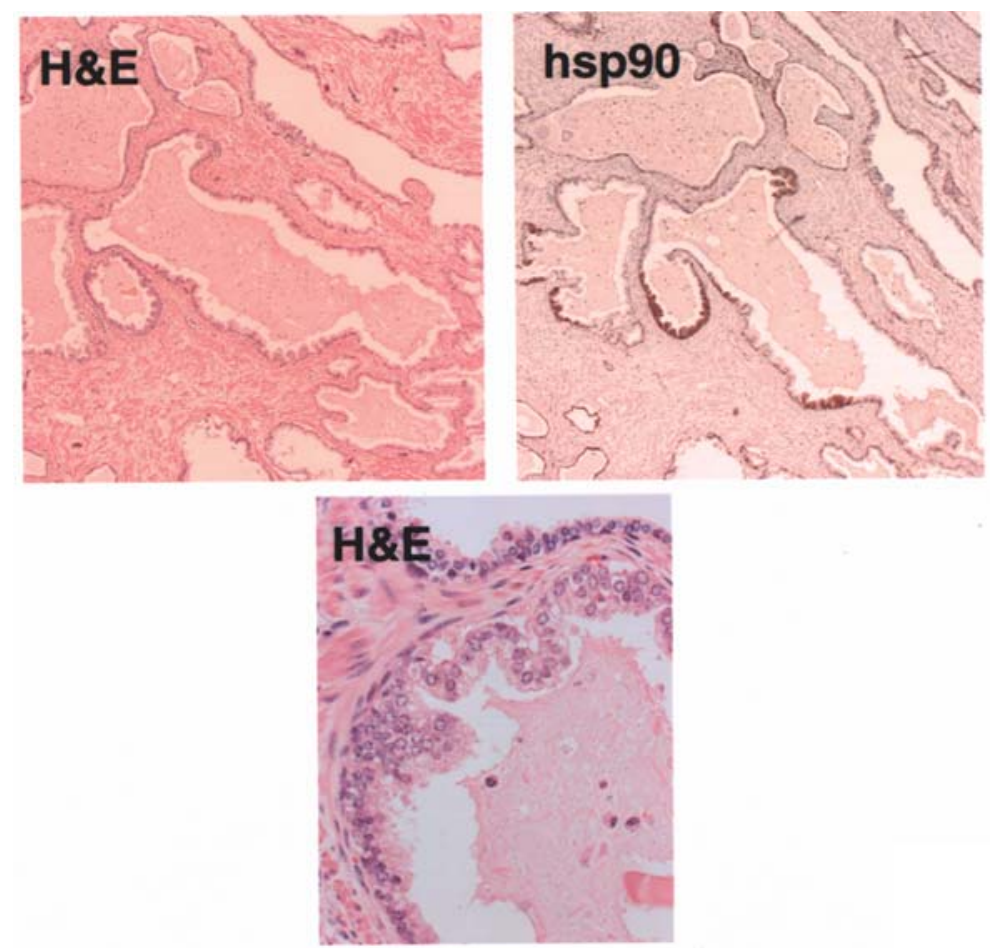

Figure 3. hsp90 immunostaining as a tool to detect high grade PIN. High grade PIN is considered the most likely precursor to prostate cancer. The characteristic enlarged nuclei and nucleoli of high grade PIN are evident under high power with H\&E (lower panel). On low power (H\&E upper left), PIN is easily detected with hsp90 immunostaining (upper right panel).

\section{Discussion}

Here we demonstrate that hsp90 and p23 levels are typically low to undetectable in epithelial cells comprising BPH but consistently expressed at high to moderate levels in carcinomas, regardless of the histologic subtype. We have analyzed
50 PIN and found that, like the carcinomas, 96\% express hsp90 and 94\% express p23. This, coupled with a lack of correlation between Gleason score and staining intensity, indicates that elevated levels of these chaperones are a relatively early, but stable, event during prostate carcinogenesis. Previous studies have similarly reported that hsp90 
expression does not correlate with Gleason score or other clinocopathological parameters including neural invasion, surgical margins or lymph node metastasis $(12,13)$. Widespread expression of hsp90 and p23 in PIN and carcinomas suggests their limited utility as markers to aid in risk stratification. Since our study was confined to high grade in situ lesions, we cannot discount the possibility that lower levels of hsp90 and p23 are not expressed in low grade PIN. Nevertheless, our data clearly show that the hsp90 chaperone complex is consistently differentially expressed in PIN compared to BPH; therefore, we propose that hsp90 immunostaining could minimize the likelihood that a small focus of intraepithelial neoplasia is overlooked in a prominent microscopic field of hyperplasia (as illustrated in Fig. 3). We also show that hsp90 and p23 are abundantly expressed in several normal prostatic constituents, a finding highly consistent with the hsp90 chaperone complex having a repertoire of client proteins implicated in normal cellular processes (7) and chaperone-mediated assembly occurring predominantly in close association with the endoplasmic reticulum (17).

If the regulation of telomerase activity during prostate cancer progression involves chaperone-mediated assembly and hsp90 and p23 remain stably associated with the telomerase holoenzyme, as our previous in vitro data indicate $(6,10)$, we reasoned that the hsp90 chaperone complex would be detectable in the nuclei of telomerase expressing prostate cancer cells in clinical specimens. Although telomerase activity is readily detectable in human cancer specimens using a PCR-based (TRAP) assay $(1,11,18)$, it is often a challenge to detect hTERT protein in situ presumably due to a low copy number of mRNA transcripts. However, we observed nuclear hTERT in all 9 prostate carcinoma cases exhibiting nuclear hsp90 (6/9) and/or p23 (9/9). Moreover, those cancers with nuclear hsp90 chaperone complex had a higher combined Gleason score (6.9 versus 6.5 for the entire case set of 60), thus establishing a correlation between nuclear hsp90 chaperone complex and telomerase in moderate-to-high grade prostate carcinomas.

We consistently observed intense hTERT immunostaining in basal cells lining glandular structures associated with hyperplasia and PIN, as previously reported (15). Basal cells also ubiquitously co-expressed hsp90 and p23. Since the current study was limited to archival tissue, telomerase activity levels could not be assessed. But if basal cells express telomerase, as suggested by their expression patterns for hTERT, hsp90 and p23, perhaps low levels of telomerase in 'normal' tissue adjacent to prostate cancer reflects basal cells rather than local invasion of micro-metastasis, as previously proposed (1). Expression of hTERT, hsp90 and p23 is also consistent with chaperone-mediated assembly of telomerase in normal cells including basal cells, which have been implicated as a stem cell compartment in the prostate (19).

Hsp90 inhibition strategies are currently being tested in clinical trials $(8,9)$. Theoretically, disrupting hsp90-client protein complexes involved in telomere maintenance and multiple growth signaling pathways would be highly effective in managing a variety of tumor types. However, our data show that in the prostate high levels of hsp90 and p23 proteins are not unique to in situ lesions and malignant tissues. Since hsp90 is expressed in many normal prostatic consti- tuents, one would predict that chaperone inhibition strategies would adversely affect normal prostate cell function, unless pharmacologic agents could be designed to selectively target hsp90-interactions in cancer cells rather than globally targeting the hsp90 chaperone complex.

\section{Acknowledgements}

We are grateful to Dr David Toft (Mayo Graduate School, Rochester, MN) for generously providing the hsp90 and p23 monoclonal antibodies and to Yvonne Veloso in Anatomic Pathology Division of the Department of Pathology at Virginia Commonwealth University for assisting with the automatic immunostaining. This study was supported by a grant from the Department of Defense (DAMD17-02-1-0152).

\section{References}

1. Shay JW and Bacchetti S: A survey of telomerase activity in human cancer. Eur J Cancer 5: 787-791, 1997.

2. Feng J, Funk WD, Wang S-S, et al: The RNA component of human telomerase. Science 269: 1236-1241, 1995.

3. Meyerson M, Counter CM, Eaton EN, et al: hEST2, the putative human telomerase catalytic subunit gene, is upregulated in tumor cells and during immobilization. Cell 90: 785-795, 1997.

4. Nakamura TM, Morin GB, Chapman KB, et al: Telomerase catalytic subunit homologs from fission yeast and human. Science 277: 955-959, 1997

5. Holt SE, Aisner DL, Baur J, et al: Functional requirement of p23 and Hsp90 in telomerase complexes. Genes Dev 13: 817-826, 1999.

6. Forsythe HL, Jarvis JL, Turner JW, et al: Stable association of hsp90 and p23, but not hsp70, with active human telomerase. J Biol Chem 276: 15571-15574, 2001.

7. Neckers L and Ivy SP: Heat shock protein 90. Curr Opin Oncol 15: 419-426, 2003.

8. Goetz MP, Toft DO, Ames MM and Erlichman C: The Hsp90 chaperone complex as a novel target for cancer therapy. Ann Oncol 14: 1169-1176, 2003.

9. Neckers L: Hsp90 inhibitors as novel cancer chemotherapeutic agents. Trends Mol Med 8: S55-S61, 2002.

10. Akalin A, Elmore LW, Forsythe HL, et al: A novel mechanism for chaperone-mediated telomerase regulation during prostate cancer progression. Cancer Res 61: 4791-4796, 2001

11. Zhang W, Kapusta LR, Slingerland JM, et al: Telomerase activity in prostate cancer, prostatic intraepithelial neoplasia, and benign prostatic epithelium. Cancer Res 58: 619-621, 1998.

12. Kurahashi T, Miyake H, Hara I and Fujisawa M: Expression of major heat shock proteins in prostate cancer: correlation with clinicopathological outcomes in patients undergoing radical prostatectomy. J Urol 177: 757-761, 2007.

13. Zellweger T, Ninck C, Bloch M, et al: Expression patterns of potential therapeutics targets in prostate cancer. Int J Cancer 113: 619-628, 2005.

14. Felt SJ and Toft DO: p23, a simple protein with complex activities. Cell Stress Chaperones 8: 108-113, 2003

15. Krebs J, Saremaslani P and Caduff R: ALG-2: a $\mathrm{Ca}^{2+}$-binding modulator protein involved in cell proliferation and in cell death. Biochim Biophysica Acta 1600: 68-73, 2002.

16. Iczkowski KA, Pantazis G, McGregor DH, et al: Telomerase reverse transcriptase subunit immunoreactivity. Cancer 95: 2487-2493, 2002.

17. Ma Y and Hendershot LM: ER chaperone functions during normal and stress conditions. J Chem Neuroanat 28: 51-65, 2004.

18. Wymenga LFA, Wisman GBA, Veenstra R, et al: Telomerase activity in needle biopsies from prostate cancer and benign prostates. Eur J Clin Invest 30: 330-335, 2000.

19. Lam JS and Reiter RE: Stem cells in prostate and prostate cancer development. Urol Oncol 24: 131-140, 2006. 